EXTENDED REPORT

\title{
Evaluation of the measurement of refractive error by the PowerRefractor: a remote, continuous and binocular measurement system of oculomotor function
}

\author{
O A Hunt, J S Wolffsohn, B Gilmartin
}

Br J Ophthalmol 2003;87:1504-1508

See end of article for authors' affiliations

Correspondence to: Dr J S Wolffsohn, School of Life and Health Sciences, Optometry, Aston University, Aston Triangle, Birmingham B4 7ET, UK; i.s.w.wolffsohn@ aston.ac.uk

Accepted for publication 17 March 2003
Background/aim: The technique of photoretinoscopy is unique in being able to measure the dynamics of the oculomotor system (ocular accommodation, vergence, and pupil size) remotely (working distance typically 1 metre) and objectively in both eyes simultaneously. The aim of this study was to evaluate clinically the measurement of refractive error by a recent commercial photoretinoscopic device, the PowerRefractor (PlusOptiX, Germany).

Method: The validity and repeatability of the PowerRefractor was compared to: subjective (noncycloplegic) refraction on 100 adult subjects (mean age 23.8 (SD 5.7) years) and objective autorefraction (Shin-Nippon SRW-5000, Japan) on 150 subjects (20.1 (4.2) years). Repeatability was assessed by examining the differences between autorefractor readings taken from each eye and by re-measuring the objective prescription of 100 eyes at a subsequent session.

Results: On average the PowerRefractor prescription was not significantly different from the subjective refraction, although quite variable (difference $+0.05(0.63) D, p=0.41$ ) and more negative than the SRW5000 prescription (by $-0.20(0.72) \mathrm{D}, \mathrm{p}<0.001)$. There was no significant bias in the accuracy of the instrument with regard to the type or magnitude of refractive error. The PowerRefractor was found to be repeatable over the prescription range of $-8.75 \mathrm{D}$ to $+4.00 \mathrm{D}$ (mean spherical equivalent) examined.

Conclusion: The PowerRefractor is a useful objective screening instrument and because of its remote and rapid measurement of both eyes simultaneously is able to assess the oculomotor response in a variety of unrestricted viewing conditions and patient types.
$\mathrm{T}$ he oculomotor triad comprising ocular accommodation, vergence, and pupil size is essential to seeing a clear, single, luminance intensity controlled image of the outside world. Continuous measurement of the oculomotor system is needed to understand further conditions such as ocular neurological effects and their progression, the potential of multifocal intraocular lenses, and the efficacy of orthoptic training. Although many techniques have been devised to measure accommodation continuously over the past two decades, photoretinoscopy is unique in enabling the measurement of accommodation, vergence, and pupil size in both eyes simultaneously, objectively, remotely (typically the camera is placed 1 metre from the eyes) and continuously. ${ }^{1}$ The PowerRefractor is the first commercially available instrument to use photoretinoscopy and allow continuous measurement of the oculomotor triad. The instrument is marketed principally as a vision screener, for the objective measurement of refractive error.

Choi and colleagues ${ }^{2}$ attempted to validate the PowerRefractor, but there were several limitations to their study: the PowerRefractor measures were compared in only 15 subjects with the patient's previous spectacle prescription and not in a current full subjective refraction; subjective refraction was performed on 40 patients, but details of the refraction were not given and the subjects had a variety of ocular pathologies; the authors' kindergarten study only compared the results with the Canon R-1 open field autorefractor; the intrasession repeatability was not assessed and intersession repeatability was only measured in the kindergarten group.

Three different techniques of photorefraction have been described: orthogonal, ${ }^{3}$ isotropic, ${ }^{4}$ and eccentric, ${ }^{56}$ but all work on the principal of analysing the vergence of reflected light rays returning to a camera after illuminating a point on the retina. The PowerRefractor uses the latter technique, with an infrared light source located on the edge of a mask, eccentric to the optical axis of the camera. If the eye is accurately focused at the camera distance, the infrared light reflected from the fundus is imaged in the camera plane as an even luminance over the entire pupil. However, if the eye is defocused myopically with respect to the camera, the light reflected from the fundus is focused in a plane between the eye and the camera and subsequently spread out in a cone, the angle of which depends on the amount of defocus. Because the mask covers part of the camera lens, only rays emanating from same part of the pupil are imaged. Conversely, for an eye focused hyperopically in respect of the camera, only rays emanating from the opposite part of the pupil will be illuminated. The resulting light gradient across the pupil is measured by the PowerRefractor in six meridians separated by $30^{\circ}$ simultaneous to a pulse of infrared light emitting diodes (LEDs) mounted on a knife edge perpendicular to each meridian in turn. The gradient of each pair of opposite meridians is averaged to remove the effect of asymmetrical meridians and the spherocylindrical refraction calculated. ${ }^{78}$

The aim of this study was to assess the validity and repeatability of the PowerRefractor to measure refractive error in non-cyclopleged adults to both subjective refraction and to a recently validated open field autorefractor, the ShinNippon SRW-5000.

\section{PATIENTS AND METHODS}

In all, 100 subjects ( 40 male and 60 female), with a mean age of 23.8 (SD 5.7) years (range from 18 to 45 years, median 22.0 years) participated in the study. Subjects with ocular 
pathologies and abnormal binocular vision were excluded from the study. Standard subjective refraction, using an end point criterion of maximum plus consistent with best vision, was performed on 200 eyes after the purpose of the study was explained and informed consent given. The study had previous approval by the institutional human science ethics committee. All refractions were performed by a UK trained and registered optometrist, who was masked as to the patient's habitual prescription and the results of the autorefraction. Retinoscopy was performed initially on all subjects followed by cross cylinder to locate the axis of the cylinder (in $2.5^{\circ}$ increments) and its power (in 0.25D increments). Best sphere, duochrome and binocular balancing (Humphris technique) was used to refine the spherical component power (in $0.25 \mathrm{D}$ increments).

Autorefraction was carried out with the PowerRefractor (located 1 metre from the subject) in full refraction mode by a second optometrist. Subjects were seated such that both eyes were visible in the instrument monitor and viewed the camera lens (as specified in the manufacturer's instructions). A series of 50 readings were taken in quick succession (taking on average 10-12 seconds) from each eye and the final autorefractor prescription calculated from the average of the last five valid (identified by green coloration) results (power in increments of $0.25 \mathrm{D}$, cylindrical component axis to $<0.1^{\circ}$ ). The PowerRefractor readings were taken under an ambient illumination of 39.6 lux, which ensured that no readings were rejected because the pupil size was too small. Measurements of repeatability were obtained by examining the differences between the five readings taken from each eye and by remeasuring the prescription of 100 eyes at a subsequent session at the same time of day, 7-10 days after the first readings were taken. The validity of autorefractors is traditionally assessed by comparing their results with that of subjective refraction. ${ }^{9}$ This is because they are principally designed to assist ophthalmologists and optometrists to reach the end point of subjective refraction as quickly as possible, in a manner similar to that of retinoscopy

The PowerRefractor was also compared to another commercially available autorefractor, the Shin-Nippon SRW-5000 in a separate cohort of 150 subjects aged 18-37 years (average 20.1 (SD 4.2) years, median 19.0 years), $45 \%$ of whom were male. The Shin-Nippon SRW-5000 is an infrared open view autorefractor found to be valid and repeatable compared to subjective refraction in both adults and children. ${ }^{10}{ }^{11}$ Subjects were positioned such that the eye under test viewed a Maltese cross at 5 metres, so that the instrument was directly aligned with the visual axis of the eye. Five readings were taken in quick succession (power in increments of $0.125 \mathrm{D}$, cylindrical component axis to $1^{\circ}$ ). PowerRefractor measures were taken by another optometrist, as described above.

\section{Statistical analysis}

As the refractive error of the two eyes are related, only data from the right eye were analysed. The bias between measures (the mean difference, standard deviation, and 95\% confidence limits) were calculated and presented graphically. ${ }^{12}$ Comparison between measures were performed using paired two tailed $t$ tests. Assessing the variance in the astigmatic component poses a problem in the conventional clinical notation. ${ }^{9}$ Therefore the sphere, cylinder, and axis component were converted into a vector representation for analysis ${ }^{13}$ : a spherical lens of power MSE (the mean spherical equivalent $=$ sphere $+[$ cylinder $/ 2]) ;$ Jackson cross cylinder at axis $0^{\circ}$ with power $\mathrm{J}_{0}(=-[$ cylinder $/ 2] \cos [2 \times$ axis $])$; Jackson crosscylinder at axis $45^{\circ}$ with power $\mathrm{J}_{45} \quad(=-$ [cylinder/2] $\sin [2 \times$ axis]).

\section{RESULTS}

The refractive error of the sample, as represented by the subjective refraction, ranged from -8.75 to $+4.00 \mathrm{D}$ mean spherical equivalent (MSE mean $=-1.44(\mathrm{SD} 2.44) \mathrm{D})$. The maximum amount of measured astigmatism was $-4.50 \mathrm{DC}$.

Prescription component differences between the PowerRefractor and subjective and objective SRW-5000 refraction are shown in Table 1 . The mean difference in components measured by the PowerRefractor were in better agreement with non-cycloplegic subjective refraction than the SRW-5000 autorefractor, but were variable in both cases (Figs 1 and 2). The broken lines on the graphs (95\% confidence interval) indicate the extent to which the PowerRefractor might over-read or under-read compared to the alternative methods examined (that is, the PowerRefractor might read as much as $1.28 \mathrm{D}$ above or 1.18D below subjective refraction for MSE).

There appears to be no bias in the accuracy of the PowerRefractor with the type or magnitude of refractive error (slope of regression line $<0.05$ ), although there are a few outlying values to the data, principally those with high myopia. Approximately 39\% of PowerRefractor readings were within $\pm 0.25 \mathrm{D}$, and $67 \%$ within $\pm 0.50 \mathrm{D}$ of the MSE as found by subjective refraction and $31 \%$ were within $\pm 0.25 \mathrm{D}$, and $57 \%$ within $\pm 0.50 \mathrm{D}$ of the MSE as found by the SRW5000 autorefractor (Fig 1B).

Figure 2 shows the difference in the $\mathrm{J}_{0}$ and $\mathrm{J}_{45}$ component of patients' refractions between subjective refraction, SRW-5000 autorefractor and PowerRefractor compared to the mean value. Approximately 50\% of PowerRefractor measures were within $\pm 0.25 \mathrm{D}$ and $74 \%$ within $\pm 0.50 \mathrm{D}$ of the cylindrical component of the prescription found by subjective refraction and $49 \%$ were within $\pm 0.25 \mathrm{D}$ and $81 \%$ within $\pm 0.50 \mathrm{D}$ of the cylindrical component found by SRW5000 autorefraction. The axis of the cylindrical component was less reliable than the spherical and cylindrical power components (Table 2). However, if only cylindrical components $\geqslant 0.75 \mathrm{D}$ are considered (as these are likely to have more than a $0.1 \log$ MAR affect on distance visual acuity if significantly off axis), the accuracy of cylindrical axis calculated by the PowerRefractor is found to be improved.

Intersession repeatability (that is, the standard deviation of differences between five measures taken on one occasion)

Table 1 Mean difference (SD) in refractive components between the PowerRefractor and non-cycloplegic subjective or SRW-5000 autorefractor objective refraction

\begin{tabular}{|c|c|c|c|c|}
\hline \multirow{2}{*}{$\begin{array}{l}\text { Refractive } \\
\text { component }\end{array}$} & \multicolumn{2}{|c|}{ PowerRefractor $v$ subjective refraction } & \multicolumn{2}{|c|}{ PowerRefractor v Shin-Nippon SRW-5000 } \\
\hline & Mean difference (D) & Significance & Mean difference (D) & Significance \\
\hline $\begin{array}{l}\text { MSE } \\
\text { Sphere } \\
J_{0} \\
J_{45} \\
\text { Cylinder }\end{array}$ & $\begin{array}{l}+0.05(0.63) \\
+0.17(0.56) \\
+0.09(0.28) \\
-0.02(0.15) \\
-0.24(0.52)\end{array}$ & $\begin{array}{r}0.41 \\
<0.01 \\
<0.01 \\
0.15 \\
<0.001\end{array}$ & $\begin{array}{l}-0.20(0.72) \\
-0.14(0.77) \\
+0.11(0.31) \\
-0.02(0.18) \\
-0.11(0.58)\end{array}$ & $\begin{array}{l}<0.001 \\
<0.05 \\
<0.001 \\
0.23 \\
<0.05\end{array}$ \\
\hline
\end{tabular}



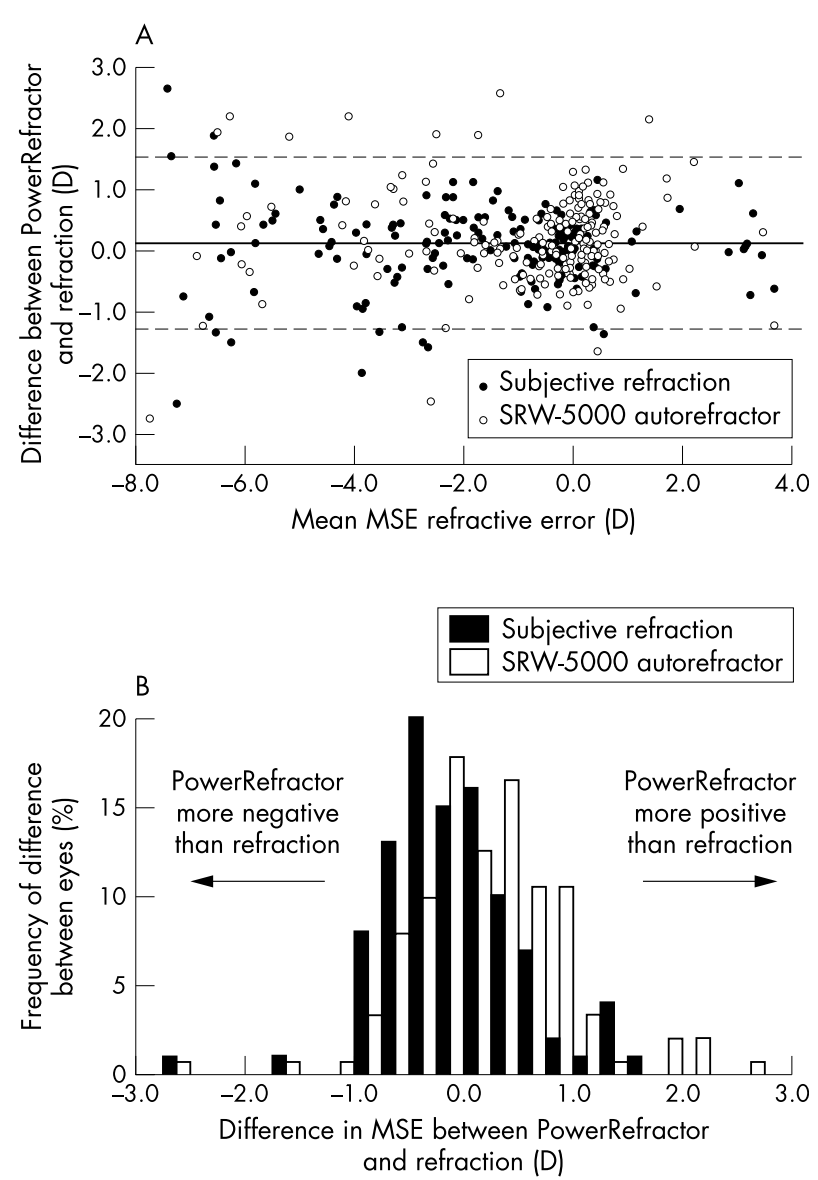

Figure 1 Difference between PhotoRefractor measures and subjective or SRW-5000 autorefractor objective MSE refraction. $N=100 / 150$ eyes.

was $0.050 \mathrm{D}$ for the spherical component, $0.062 \mathrm{D}$ for the cylindrical component $0.062 \mathrm{D}$ for the mean spherical equivalent, 0.036 for the $\mathrm{J}_{0}$ and 0.029 for the $\mathrm{J}_{45}$ component. Table 3 presents the mean intrasession differences between the prescription components. These were found to be small, with the majority of the second visit prescriptions falling within plus or minus $0.50 \mathrm{D}$ of the initial measurement.

\section{DISCUSSION}

The ability of the PowerRefractor to measure continuously from a remote location, binocular parameters of the complete oculomotor triad, will prove to be of great value in optometric and ophthalmological research. Commercial photorefractive devices have been available for a number of years, but only for static measure of refractive error. For example the MTI

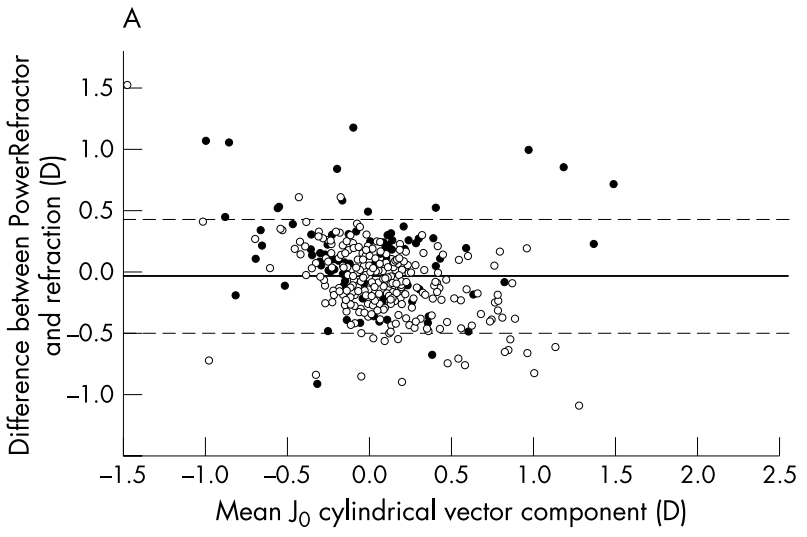

B

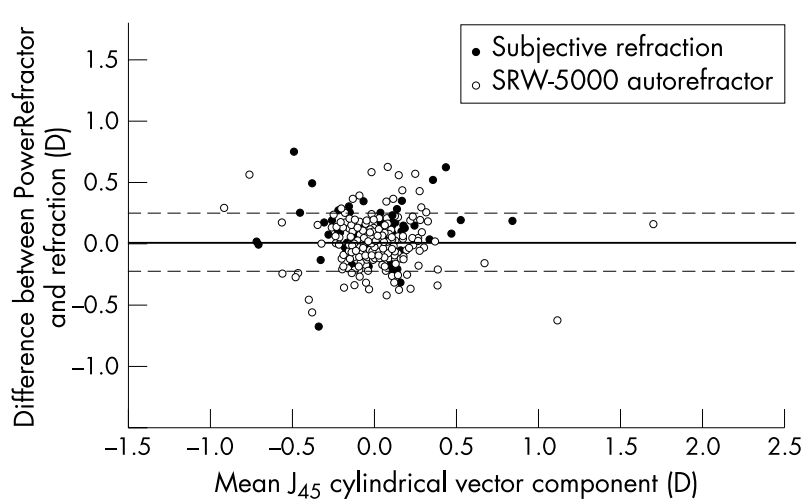

Figure 2 Difference between PhotoRefractor measures and subjective or SRW-5000 autorefractor objective $\mathrm{J}_{0}$ and $\mathrm{J}_{45}$ vector components of refraction. $\mathrm{N}=100 / 150$ eyes.

photoscreener is used in preschool screening in the United States, but requires the Polaroid of the imaged pupil crescents to be measured individually by a trained observer. ${ }^{14}$ Schaeffel and Howland ${ }^{15}$ tried to digitise this process, but were limited by the processor speed at the time, the dead zone obscuring low prescriptions and the non-linearity of the brightness across the pupil. The TOMEY ViVA and PR2000 were found to have only reasonable accuracy up to $\pm 3.00 \mathrm{D},{ }^{16}{ }^{17}$ which may be the result of using a single light source, resulting in a large dead zone and a non-linear intensity gradient versus refractive error. ${ }^{1}$ Linearity is improved by using LEDs at more than one eccentricity below the knife edge ${ }^{18}$ and by measuring the slope of the brightness profile across the pupil rather than the height of the pupil crescents. ${ }^{19}$

Although on average the PowerRefractor prescription was similar to that of subjective refraction, the range of differences was large (up to $3.5 \mathrm{D}$ ), with $95 \%$ of readings

Table 2 Comparison of the axis of the cylindrical component measured by the PowerRefractor with both non-cycloplegic subjective and SRW-5000 autorefractor objective refraction

\begin{tabular}{|c|c|c|c|c|}
\hline \multirow[b]{2}{*}{ PowerRefractor $v$} & \multicolumn{2}{|c|}{$\begin{array}{l}\text { All prescriptions with a cylindrical } \\
\text { component (\%) }\end{array}$} & \multicolumn{2}{|c|}{$\begin{array}{l}\text { Prescriptions with a cylindrical component } \\
\text { (subjective) } \geqslant 0.75 \mathrm{D}(\%)\end{array}$} \\
\hline & Subjective & SRW-5000 & Subjective & SRW-5000 \\
\hline No of subjects & 74 & 124 & 30 & 37 \\
\hline Within $\pm 5^{\circ}$ & 32 & 23 & 40 & 41 \\
\hline Within $\pm 10^{\circ}$ & 54 & 37 & 67 & 59 \\
\hline Within $\pm 15^{\circ}$ & 65 & 50 & 80 & 73 \\
\hline Within $\pm 20^{\circ}$ & 70 & 56 & 87 & 76 \\
\hline
\end{tabular}


Table 3 Difference in refractive component of the PowerRefractor found between different sessions

\begin{tabular}{lllllc}
\hline & MSE & $\mathrm{J}_{0}$ & $\mathrm{~J}_{45}$ & Sphere & Cylinder \\
\hline Mean difference & 0.06 & -0.001 & 0.003 & 0.07 & -0.01 \\
SD of differences & 0.36 & 0.11 & 0.05 & 0.36 & 0.12 \\
Within $\pm 0.25 \mathrm{D}(\%)$ & 84 & - & - & 86 & 92 \\
Within $\pm 0.50 \mathrm{D}(\%)$ & 96 & - & - & 96 & 100 \\
Within $\pm 0.75 \mathrm{D}(\%)$ & 98 & - & - & 98 & 100 \\
\hline
\end{tabular}
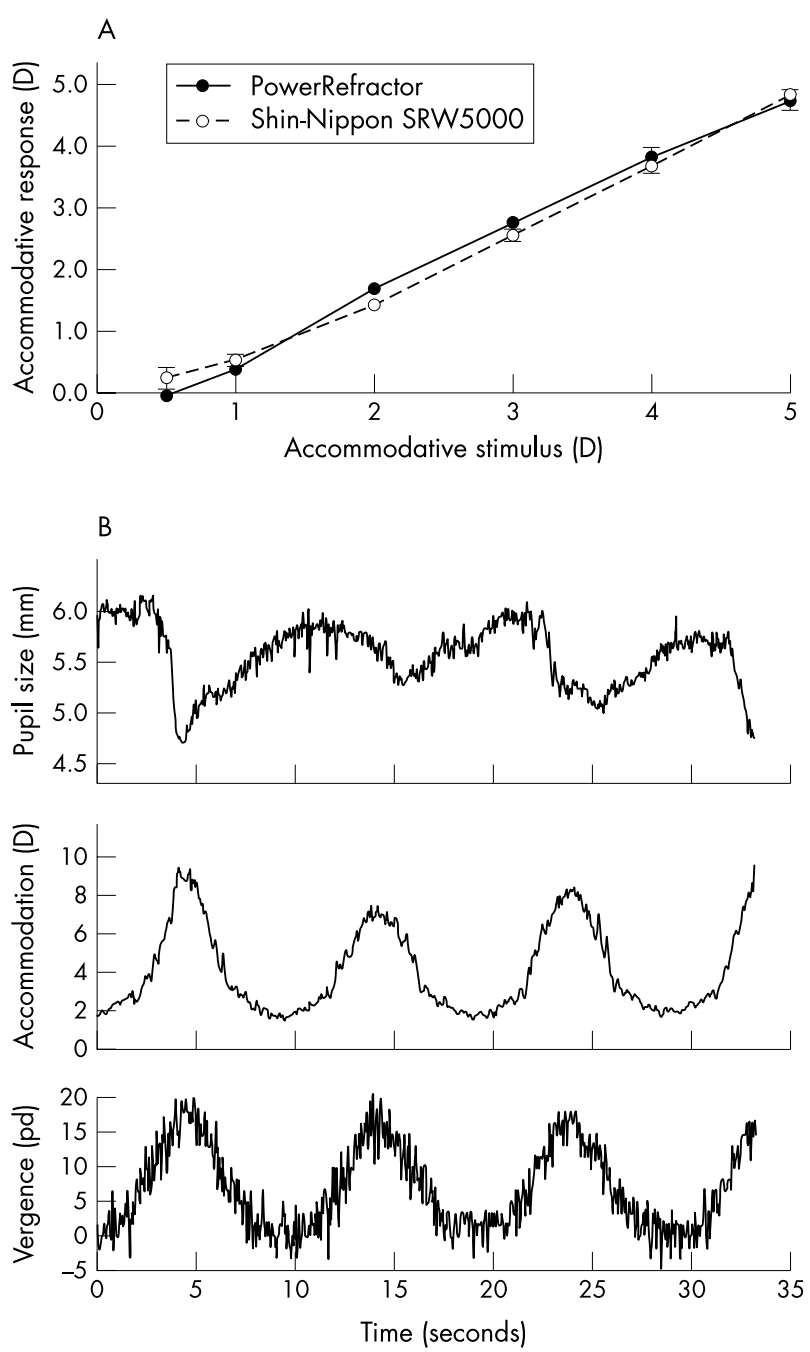

Figure 3 (A) Accommodative stimulus response curve for an emmetropic subject measured with the PowerRefractor and SRW-5000; (B) changes in the oculomotor response triad for the same subject binocularly viewing a target oscillating at $0.1 \mathrm{~Hz}$ between 10 and $50 \mathrm{~cm}$.

within $\pm 1.12 \mathrm{D}$ (Fig 2 ). This was particularly the case with myopic prescriptions over $-4.0 \mathrm{D}$, confirming the stated accuracy range of the PowerRefractor by Choi and colleagues. ${ }^{2}$ However, the discrepancy between the PowerRefractor prescription and subjective refraction within this range could still be up to $2.5 \mathrm{D}$. Undercorrecting myopia by such a degree would severely reduce a patient's visual acuity and in young children could lead to impaired visual development. Two subjects with a prescription of greater than -10.0D were assessed outside of the study, for whom the PowerRefractor indicated a prescription of only -3.0 to $-4.0 \mathrm{D}$. Compared to the Shin-Nippon SRW-5000 autorefractor, the PowerRefractor prescription was more negative and again had a wide range of differences. Both autorefractors measure refractive error using image analysis techniques of infrared light reflected from the fundus, although the PowerRefractor measures the light gradient and the SRW-5000 the reflected image size. Compared to previously validated open field autorefractors (for example, the Canon R-1 and Shin-Nippon SRW5000) the PowerRefractor is, on average, just as accurate, but approximately $24-42 \%$ more variable. ${ }^{10} 20$

Inter-session and intra-session repeatability of the PowerRefractor was found to be small and was similar to previously validated open field autorefractors. ${ }^{10} 20$ This would indicate that the variability is due to the lack of individual calibration, the poor accommodative stimulus of the camera head LEDs, and perhaps the linear range of the change in light gradient across the pupil with refractive error, ${ }^{19}$ rather than stability of the imaging technique. An emmetrope focusing accurately at the camera distance should have a uniformly illuminated pupil. However, chromatic aberration, lead of accommodation, and in children small eye artefacts, all combine to produce focusing error and hence a light gradient across the pupil. As a slope change of +0.50 translates to approximately 1.0D, small changes in slope can have a significant effect of apparent refraction. In addition, as the camera head is closer than optical infinity, a $\leqslant-1.0 \mathrm{D}$ myope, emmetrope and pre-presbyopic hypermetrope could all appear to have the same refractive error when focusing on the camera head LEDs at 1 metre. A more suitable target would be a non-accommodative distant target (such as a spot of light) with +1.0DS added to the internally generated prescription.

Figure 3 demonstrates the accommodative stimulus response curve for an emmetropic subject measured with the PowerRefractor and SRW-5000 and changes in the oculomotor triad when binocularly viewing a target oscillating sinusoidally at $0.1 \mathrm{~Hz}$ between $10 \mathrm{~cm}$ (10D) and $50 \mathrm{~cm}$ (2D) from the subject. The stimulus response curve shows the accuracy of the technique compared to a well validated instrument and the trace to an oscillating target, the potential for the PowerRefractor to be a valuable tool in ophthalmological research.

In conclusion, the PowerRefractor is a practical and effective autorefractor over the range of refractive error $(-8.75$ to $+4.00 \mathrm{D})$ and age ( $18-45$ years) assessed. It is likely to have a particular use in the screening of children and patients unable to give a subjective response. The variability, particularly with high myopia is, however, of some concern, with potential under correction of over $1 \mathrm{D}$ in $\sim 7 \%$ of cases. The instrument will also be useful in research as it is able to measure remotely accommodation, pupil size, and gaze direction in both eyes simultaneously at $25 \mathrm{~Hz}$, providing objective measures of the oculomotor system while subjects binocularly view a natural scene in an unenclosed and unrestricted environment. 


\section{ACKNOWLEDGEMENTS}

Information on the workings of the PowerRefractor was kindly provided by Frank Schaeffel. The authors have no proprietary interests in the PowerRefractor.

\section{Authors' affiliations}

O A Hunt, J S Wolffsohn, B Gilmartin, Neurosciences Research Institute, School of Life and Health Sciences, Aston University, Birmingham, UK

\section{REFERENCES}

1 Wolffsohn JS, Hunt OA, Gilmartin B. Continuous measurement of accommodation in human factor applications. Ophthalmic Physiol Opt 2002;22:380-4.

2 Choi M, Weiss S, Schaeffel F, et al. Laboratory, clinical, and kindergarten test of a new eccentric infrared photorefractor (PowerRefractor). Optom Vis $\mathrm{Sci}$ 2000;77:537-48.

3 Howland HC, Howland B. Photorefraction: a technique for study of refractive status at distance. J Opt Soc Am 1974;64:240-9

4 Howland $\mathrm{H}$, Braddick $\mathrm{O}$, Atkinson J, et al. Optics of photorefraction: orthogonal and isotropic methods. J Opt Soc Am 1983;73:1701-8.

5 Bobier WR, Bradick OJ. Eccentric photorefraction: Optical analysis and empherical measures. Am J Optom Physiol Opt 1985;62:614-20.

6 Howland HC. Optics of photoretinoscopy: results from ray tracing. Am J Optom Physiol Opt 1985:62:621-5.

7 Gekeler F, Schaeffel F, Howland HC, et al. Measurement of astigmatism by automated infrared photoretinoscopy. Optom Vis Sci 1997:74:472-82.
8 Wesemann W, Norcia AM, Allen D. Theory of eccentric photorefraction (photoretinoscopy): astigmatic eyes. J. Opt Soc Am. (A) 1991;8:2038-47.

9 Bullimore MA, Fusaro RE, Adams CW. The repeatability of automated and clinical refraction. Optom Vis Sci 1998:75:617-22.

10 Mallen EAH, Wolffsohn JS, Gilmartin B, et al. Clinical evaluation of the ShinNippon SRW-5000 autorefractor in adults. Ophth Physiol Opt 2001;21:101-7.

11 Chat SWS, Edwards MH. Clinical evaluation of the Shin-Nippon SRW-5000 autorefractor in children. Ophth Physiol Opt 2001;21:87-100.

12 Bland JHS Altman DG. Statistical methods for assessing agreement between two methods of clinical measurement. Lancet 8476:307-10.

13 Thibos LN, Wheeler W, Horner D. Power vectors: an application of fourier analysis to the description and statistical analysis of refractive error. Optom Vis Sci 1997:74:367-75.

14 Tong PY, Bassin RE, Enke-Miyazaki E, et al. Screening for amblyopia in preverbal children with photoscreening photographs II. Sensitivity and specificity of the MTI PhotoScreener. Opthalmol 2000;107:1623-9.

15 Schaeffel F, Howland $\mathrm{H}$. Measurement of pupil size, direction of gaze and refractive state by online analysis of digitized video images. OSA 1991 Technical Digest on Non-invasive Assessment of Vision 1991;1:76-9.

16 Thompson AM, Counts R, Li T, et al. Accuracy and precision of the Tomey ViVA infrared photorefractor. Optom Vis Sci 1996;73:644-52.

17 Williams C, Lumb R, Harvey I, et al. Screening for refractive errors with the Topcon PR2000 pediatric refractometer. Invest Ophthalmol Vis Sci 2000;41:1031-7

18 Schaeffel F, Farkas L, Howland HC. Infrared photoretinoscope. Appl Optics 1987;26:1505-9

19 Schaeffel F, Wilhelm $\mathrm{H}$, Zrenner E. Inter-individual variability in the dynamics of natural accommodation in humans: relation to age and refractive errors. J Physiol 1993;461:301-20.

20 McBrien NA, Millodot M. Clinical evaluation of the Canon Autoref R-1 Am J Optom Physiol Opt 1985;62:786-92. 